\title{
Intracrine androgen biosynthesis in renal cell carcinoma
}

\author{
Geun Taek Lee ${ }^{1,5}$, Christopher S Han ${ }^{1,2,5}$, Young Suk Kwon ${ }^{1,2}$, Rutveej Patel ${ }^{2}$, Parth K Modi ${ }^{2}$, Seok Joo Kwon ${ }^{1}$, \\ Izak Faiena ${ }^{2}$, Neal Patel ${ }^{2}$, Eric A Singer ${ }^{1,2}$, Han-Jong Ahn ${ }^{3}$, Wun-Jae Kim ${ }^{4}$ and Isaac Y Kim ${ }^{*, 1,2}$ \\ ${ }^{1}$ Section of Urologic Oncology, Rutgers Cancer Institute of New Jersey, New Brunswick, NJ 08903, USA; ${ }^{2}$ Division of Urology, \\ Rutgers Robert Wood Johnson Medical School, New Brunswick, NJ 08903, USA; ${ }^{3}$ Department of Urology, Asan Medical Center, \\ Seoul 05505, Korea and ${ }^{4}$ Department of Urology, Chungbuk National University College of Medicine, Cheongju 28644, Korea
}

Background: Renal cell carcinoma (RCC) is one of the most lethal genitourinary cancers. The presence of androgen receptor (AR) in RCC has recently been shown to be associated with higher tumour stage irrespective of gender. Because the clinical context of androgens in female RCC patients is similar to that of prostate cancer patients undergoing androgen-deprivation therapy, mechanisms underlying the emergence of castration-resistant prostate cancer (CRPC) may be at play in AR-positive RCC cells. Therefore, we hypothesized that AR-positive RCC has intratumoral steroidogenesis and that anti-androgen therapy may result in tumour suppression.

Methods: Mice were injected with an AR-positive RCC cell line. When tumours became palpable, surgical castration was performed and tumour volume was measured. Using ELISA, the levels of intracellular testosterone and dihydrotesterone were measured in AR-positive human RCC cell lines. Lastly, male mice containing xenografts were treated with enzalutamide or abiraterone acetate (AA) for 3 weeks to measure tumour volume.

Results: We first observed in vivo that castration retards the growth of AR-positive RCC tumour xenograft in mice. Next, AR-positive human RCC cell lines and tissues were found to have elevated levels of testosterone and dihydrotestosterone and express key enzymes required for intracellular androgen biosynthesis. A mouse xenograft study with AR-positive RCC cell line using the commonly used anti-androgen therapies showed significant tumour suppression $(P<0.01)$.

Conclusions: Intracrine androgen biosynthesis is a potential source of androgen in AR-positive RCC and that the androgen signaling axis is a potential target of intervention in RCC.

Kidney cancer accounts for $\sim 5$ and $3 \%$ of new cancer cases in men and women, respectively, in the United States with 14240 estimated deaths alone in 2016 (Siegel et al, 2016). This estimate largely reflects clear cell renal cell carcinoma (RCC), which comprises the majority of kidney cancers (Hirsch et al, 2015). RCC remains one of the most lethal genitourinary cancer with a 5-year overall survival (OS) rate of 74\% (Siegel et al, 2016). In addition, $\sim 25 \%$ of patients with RCC have metastatic disease at diagnosis, and $30 \%$ of patients who undergo nephrectomy for clinically localized RCC relapse eventually (Shek et al, 2012). Although the introduction of targeted therapies has revolutionized the treatment of metastatic and recurrent RCC (mRCC), the prognosis for patients with advanced stage RCC remains poor. Therefore, it is crucial to improve our understanding of the disease process and identify new targets of intervention.

Although the prognostic value of androgen receptor (AR) expression in RCC remains controversial (Noh et al, 2013; He et al, 2014; Zhu et al, 2014; Ha et al, 2015; Zhao et al, 2016), we have demonstrated that increased expression of AR mRNA in RCC is associated with higher tumour stage irrespective of gender and that the treatment with dihydrotestosterone (DHT) causes proliferation of AR-positive cell lines, while treatment with enzalutamide (Enz),

${ }^{\star}$ Correspondence: Dr IY Kim; E-mail: kimiy@cinj.rutgers.edu

${ }^{5}$ These authors contributed equally to this work.

Received 27 July 2016; revised 23 January 2017; accepted 27 January 2017; published online 2 March 2017

(C) 2017 Cancer Research UK. All rights reserved 0007-0920/17 
a second-generation AR antagonist, results in reduced cell viability (Ha et al, 2015). Because the clinical context of androgens in female RCC patients is similar to that of prostate cancer patients undergoing androgen-deprivation therapy, proposed mechanisms underlying the emergence of castration-resistant prostate cancer (CRPC) may be at play in AR-positive RCC cells. In support of this concept, herein, we report that some RCC cell lines and ccRCC tissues have an intracellular androgen biosynthesis pathway, and castration delays the growth of tumour xenografts derived from the androgen-sensitive human RCC cell line, Caki2. Simultaneously, we have observed that Enz and AA dramatically reduce the tumorigenic potential of Caki2 tumour xenografts in mice.

\section{MATERIALS AND METHODS}

Cell lines. The human prostate cancer cell line, LNCaP and RCC cell lines, Caki1, Caki2, ACHN, A498, and Hs891.T, were purchased from American Type Culture Collection (Manassas, VA, USA). Caki1, Caki2, A498, and ACHN were from males and Hs891.T was from a female patient. Caki1, Caki2, ACHN, and Hs891.T were maintained in Dulbecco's modified eagle medium. LNCaP was maintained in RPMI 1640 medium. Unless stated otherwise, all tissue culture media were supplemented with $10 \%$ fetal bovine serum (FBS) and maintained in a $5 \% \mathrm{CO}_{2}$ and $37^{\circ} \mathrm{C}$ incubator. All cell lines were routinely tested for mycoplasma contamination, and have been authenticated with short-tandem repeat (STR) analysis (ATCC).

RNA extraction and RT-PCR analysis. Total RNA was isolated from cells using $1 \mathrm{ml}$ of TRIzol reagent (ThermoFisher Scientific, Waltham, MA, USA) using the manufacturer's recommended protocol. ImProm-II Reverse Transcription System (Promega, Madison, WI, USA) was used for reverse transcription. The primer sequences for CYP11A1 were forward ( $5^{\prime}$ - GTGGAGACCACCACCTCTGT-3') and reverse (5'-TCCTCGAAGGACATCTT GCT-3'), for CYP17A1 forward (5'-GTGGAGACCACCACCTC TGT-3') and reverse (5'- GCTGAAACCCACATTCTGGT-3'), for HSD3B1 forward (5'- CAGATGACACGCCTCACCAA-3') and reverse (5' - AGGGCGGTCGATAGGTGTAA-3'), for HSD3B2 forward (5'- TCTCAGATGACACGCCTCAC- $\left.3^{\prime}\right)$ and reverse ( $5^{\prime}-$ GG GCTGAGTAGGAAGCTCAC-3'), for HSD17B3 forward (5'-ATCCAGAGCCTCATCCATTG-3') and reverse (5'-AACGCCTTGGAAG CTGAGTA-3'), for AKR1C1 forward (5'-CATGTGGCACAGAGATCCAC-3') and reverse (5'-TACTTGAGCCCTGGCTTGTT-3'), for SRD5A1 forward ( $5^{\prime}$-ATGCGAGGAGGAAAGCCTAT-3') and reverse (5'-CACTGCACAATGGCTCAAGT-3'), and for GAPDH forward ( $5^{\prime}$-TGCACCACCAACTGCTTAGC- $\left.3^{\prime}\right)$ and reverse (5'-GGC ATGGACTGTGGTCATGAG-3').

PCR amplification was performed using a Rotor Gene 6000 (Corbett Research, Mortlake, Victoria, Australia). Real-time PCR assays were carried out in micro-reaction tubes (Corbett Research) using SYBR Premix EX Taq (Takara Bio Inc., Otsu, Japan). All samples were run in triplicate. GAPDH was used as the endogenous RNA reference gene.

Androgen analysis with ELISA. All cell lines were cultured in charcoal stripped fetal bovine serum (cFBS) (ThermoFisher Scientific) prior to measuring testosterone (T) and DHT. Testosterone EIA kit (Cayman chemical, Cat No. 582701, Ann Arbor, MI, USA: Sensitivity $6 \mathrm{pg} \mathrm{ml}^{-1}$, Cross Reactivity $140 \% 19$ Nortestosterone, $100 \%$ Testosterone, $27.4 \% 5 \alpha$-dihydrotestosterone) and DHT ELISA kit (antibodies-online, Cat No. ABIN1118017, Atlanta, GA, USA: Sensitivity $14.2 \mathrm{pg} \mathrm{ml}^{-1}$, No significant cross-reactivity or interference between DHT and analogues) were used, respectively. The reactivity of testosterone EIA kit was not applicable while the reactivity of DHT ELISA kit was human. Manufacturer's recommended protocols were used.
For tissue homogenization, tissue was thoroughly rinsed in ice-cold PBS $\left(0.02 \mathrm{moll}^{-1}, \mathrm{pH} 7.0-7.2\right)$ to remove excess blood and weighed before homogenization. Tissue was then minced into small pieces and homogenized in $800 \mu \mathrm{l}$ of PBS with a glass homogenizer on ice. Then, two freeze-thaw cycles to further break down cell membranes were performed. After that, the samples were centrifuged for $15 \mathrm{~min}$ at $1500 \times \mathrm{g}$. After removed the supernatant, assay was performed immediately.

Prostate-specific antigen (PSA) luciferase. Cell lines were transfected with lipofectamine 3000 (ThermoFisher Scientific) using our previously constructed pGL3-PSA luciferase DNA (Lee et al, 2013). Cells were incubated for $24 \mathrm{~h}$ with and without varying concentrations of DHT (10 nM and $100 \mathrm{~nm}$ ). Luciferase activity was detected with the Dual Luciferase Assay kit (Promega) according to the manufacturer's recommended protocol.

Human RCC tissue analysis. Localized female human RCC tissue positive for $A R$ were obtained from our institutional tissue bank and lysed with glass homogenizer in $5000 \mu$ l protein lysis buffer. Protein concentrations were measured with BCA protein assay kit (ThermoFisher Scientific) according to the manufacturer's recommended protocol.

Cell proliferation assay. Cells were seeded at 50000 cells per well in a 24-well plate and allowed to adhere for $24 \mathrm{~h}$. The cells were then treated with indicated concentrations of AA (Medchemexpress LLC, Monmouth Junction, NJ, USA). Cells were detached and counted using a hemocytometer three days later.

Mouse xenograft studies. Animal protocol was reviewed and approved by the Institutional Animal Care and Use Committee (IACUC) prior to initiation of the xenograft study. Male nude mice 6-8 weeks of age were obtained from Jackson Laboratory (Bar Harbor, ME, USA), and $5 \times 10^{6}$ Caki2 cells were subcutaneously injected in flanks with Matrigel $(1: 1, \mathrm{v} / \mathrm{v})$. When tumours became palpable, mice were randomly assigned to sham operation and castration group. The castration group underwent surgical bilateral orchiectomy. Each group was further randomly divided into six experimental arms: the main control arm without any treatment nor vehicle administration, the treatment arms with enzalutamide (Selleck Chemicals, Houston, TX, USA), AA, or the combination of enzalutamide and AA, and the vehicle-only arms for each Enz and AA arm. Mice in the enzalutamide arms were treated with daily oral gavage of $10 \mathrm{mg} \mathrm{kg}^{-1}$ of enzalutamide (in $1 \%$ carboxymethyl cellulose, $0.1 \%$ Tween- 80 and 5\% DMSO). Mice in the AA arms were treated with daily intraperitoneal injections of $0.5 \mathrm{mmol} \mathrm{kg}^{-1}$ of AA (in $0.1 \mathrm{ml} 5 \%$ benzyl alcohol and 95\% safflower oil solution). Tumour dimensions were measured once a week for 4 weeks. Volume was calculated using the formula length $\times$ width $\times$ height $\times 0.52$. At the end of the study, mice were killed and collected tumours were assayed for the presence of the key steroidogenesis enzymes as well as T and DHT levels as described above. Blood was collected after killing via cardiac puncture.

Statistical analysis. All the numerical data were expressed as mean \pm s.d. For comparison between two groups, student's $t$-test was used. For experiments involving more than two groups, ANOVA was performed followed by a multiple range test. A $P$-value $<0.05$ was considered statistically significant. All statistical analyses were carried out using the statistical software SPSS 12.0 (SPSS Inc., Chicago, IL, USA).

\section{RESULTS}

Castration decreases the proliferation of AR-positive RCC cells. Previously, we have reported that DHT induces the proliferation of AR-positive human RCC cell lines ( $\mathrm{Ha}$ et al, 
2015). Given that the androgen signaling involves multiple pathways, we next investigated whether the conventional androgen signaling mechanism is present in RCC cells by transfecting a luciferase reporter construct containing the promoter of the prostate-specific antigen (Lee et al, 2013). The results demonstrated that luciferase activities were induced with DHT in a concentration-dependent manner in the AR-positive cell lines (Caki2 and Hs891.T; $P<0.01$ ), while no luciferase activity was seen in the AR-negative cell line, Cakil (Figure 1A). To further examine the role of androgens in RCC in vivo, we injected the flanks of twenty nude mice with the AR-positive Caki2. When tumours became palpable, surgical castration was carried out via bilateral orchiectomy in ten randomly chosen mice. Subsequently, tumour volume was measured serially. As shown in Figure 1B, the tumour growth rate of Caki2 xenografts decreased significantly compared with the sham (non-castration) control group. The gross picture of the tumours collected at the end of the study shows the significantly smaller tumours in the castration group (Figure 1C).

Intracellular testosterone and DHT levels are elevated in ARpositive RCC cell lines. Since the clinical framework of AR in females is similar to that of castration-resistant prostate cancer, we next explored the concept of intracrine androgen synthesis in RCC by measuring intracellular $\mathrm{T}$ and DHT levels with ELISA (Figure 2A). Compared with the AR-negative RCC cell lines, ACHN and Cakil, the AR-positive RCC cell lines Caki2 and Hs891.T showed significantly elevated levels of $\mathrm{T}$ and DHT $(P<0.05)$. Intracellular T and DHT concentrations in Caki2 and Hs891.T ranged 20 to $70 \%$ of those of LNCaP, a human prostate cancer cell line with a well-characterized intracrine androgen biosynthesis (Suzuki et al, 2007).

Key enzymes for steroidogenesis are expressed in AR-positive RCC cell lines. Since elevated intracellular androgen concentrations in AR-positive RCC cell lines suggest the presence of intracrine androgen biosynthesis, we next evaluated the expression levels of key enzymes involved in steroidogenesis. The results demonstrated that both Caki2 and Hs891.T expressed CYP17A1, HSD3B1/HSD3B2, HSD17B3, AKR1C3, and SRD5A1 enzymes necessary for the conversion of circulating sex steroids into androgens (Mostaghel, 2013; Figure 2B). In contrast, the AR-negative cell lines ACHN and Cakil did not express one or more of these enzymes. Importantly, CPY11A1 was not detected in any RCC cell lines, suggesting the inability of RCC cells to convert cholesterol to androgens de novo. Caki2 was also noted to express HSD3B isoform 2 only. Both isoforms, however, function as steroid dehydrogenase/isomerases, and expression of either one is sufficient to complete steroidogenesis (Payne and Hales, 2004).

Intratumoral androgen concentration remains high following castration. To determine whether the tissue culture findings can be extended in vivo, we next measured the $\mathrm{T}$ concentrations in the serum and tumour following castration after inoculating the flanks of nude mice with Caki2. In the sham group (control), the serum $\mathrm{T}$ level was $\sim 93.49 \mathrm{ng} \mathrm{ml}^{-1}$ per $\mathrm{mg}$ protein (Figure $3 \mathrm{~A}$ ). In comparison, intratumoral $\mathrm{T}$ level was approximately eight-fold higher at $\sim 781.63 \mathrm{ng} \mathrm{ml}^{-1}$ per $\mathrm{mg}$ protein. Following castration, serum $\mathrm{T}$ level dropped by roughly $98 \%$, to $14.3 \mathrm{ng} \mathrm{ml}^{-1}$ per $\mathrm{mg}$ protein. In contrast, castration decreased the intraumoral $\mathrm{T}$ level by only $\sim 36 \%$ and was nearly 35 -fold higher than the serum level. In addition, the absolute $\mathrm{T}$ level in the tumours was $499.63 \mathrm{ng} \mathrm{ml}^{-1}$ per mg protein. As for DHT, intratumoral levels were comparable between the sham and castration group; following castration, DHT concentration in tumours was above $1000 \mathrm{ng} \mathrm{ml}^{-1}$ per mg protein (Figure 3B). Simultaneously, the effect of castration on the four androgen biosynthesis enzymes was investigated. The results demonstrated that castration significantly increased the mRNA levels of three enzymes (Supplementary Figure 1). Taken together, these observations demonstrate that the Caki2 xenografts have the capacity to produce physiologically significant levels of androgens.

Androgen biosynthesis enzymes and physiologically significant androgen levels are found in human RCC tissues. To translate the findings of the cell line studies to the clinic, we assessed androgens and its biosynthesis enzymes in human RCC tissues. Both male and female human RCC tissues were analyzed. Importantly, RT-PCR demonstrated that the three female RCC specimens examined were positive for AR mRNA when compared with the negative control Caki-1 (data not shown). When the expression status of the key androgen biosynthesis enzymes were analyzed, the four enzymes detected in RCC cell lines were also found in all of the human RCC tissues examined (Figure 4A). As predicted by the in vitro study, CYP11A1 was again not detected in the clinical specimens. When intratumorral T and DHT were
A

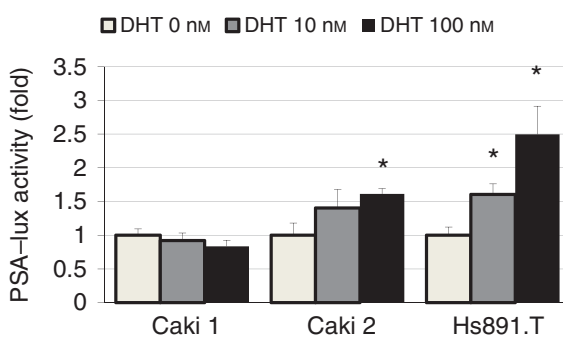

B $\rightarrow$ Castration $\rightarrow$ Non-castration

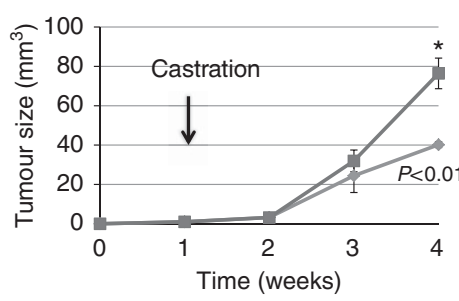

C

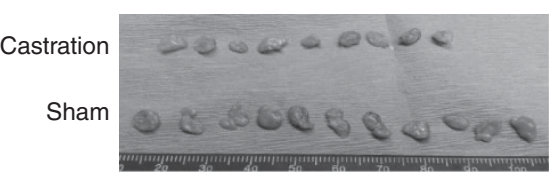

Figure 1. RCC cell lines positive for androgen receptor (AR) are sensitive to androgens. (A) Promoter activity of prostate-specific antigen (PSA) in AR-positive and -negative RCC cell lines with varying doses of DHT. Cells were transiently transfected with the reporter plasmid containing PSA promoter and luciferase. In the AR-positive cell lines, Caki2 and HS891.T, DHT increased luciferase activity in a concentration dependent manner. (B) The AR-positive RCC cell line Caki2 was injected into the flanks of 20 mice. Following the establishment of tumour, ten mice were surgically castrated and tumour volume was measured for 3 weeks. Castration decreased the tumour growth of Caki2 xenografts significantly. (C) Gross picture of tumours harvested from castration and sham control (non-castration). *statistically significant. 

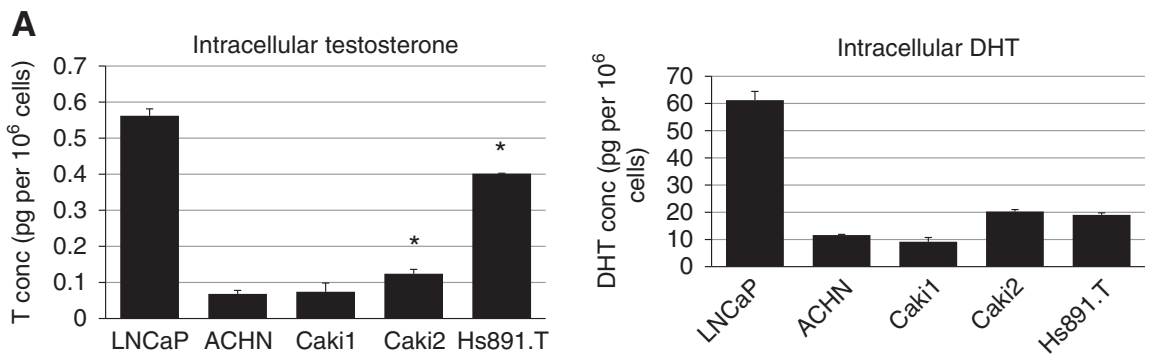

\begin{tabular}{|c|c|c|c|c|c|c|c|}
\hline & CYP11A1 & SRD5A1 & CYP17A1 & HSD17B3 & AKR1C3 & HSD3B1 & HSD3B2 \\
\hline LNCaP & + & ++ & ++ & ++ & ++ & ++ & ++ \\
\hline ACHN & - & - & - & - & - & - & - \\
\hline Hs891.T & - & + & + & + & + & + & + \\
\hline Caki1 & - & + & + & - & +++ & + & ++ \\
\hline Caki2 & - & + & + & + & +++ & - & + \\
\hline
\end{tabular}

Figure 2. AR-positive RCC cell lines have an intracrine androgen biosynthesis pathway. (A) ELISA was carried out for the intracellular testosterone (T) and dihydrotestosterone levels in the listed human RCC cell lines. Caki2 and Hs891.T are AR-positive. The human prostate cancer cell line, LNCaP, was used as the positive control. Higher levels of T and DHT were found in the AR-positive human RCC cell lines. (B) RT-PCR analysis for the expression patterns of key enzymes required for the intracellular androgen biosynthesis was carried out in each cell line. Again, LNCaP was used as the positive control. With the exception of CYP11A1, all of the key enzymes were detected in only the AR-positive human RCC cell lines, Caki2 and HS891.T. *statistically significant (with respect to ACHN).

\section{A}

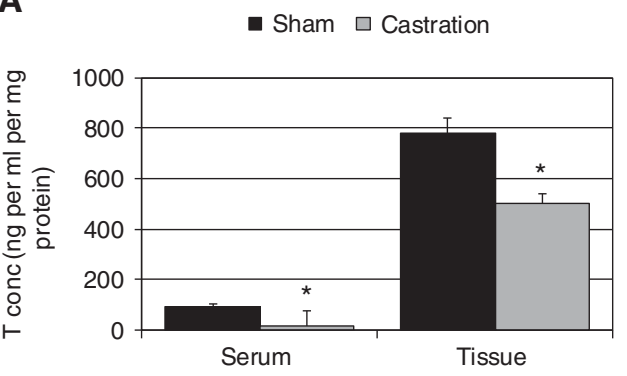

B
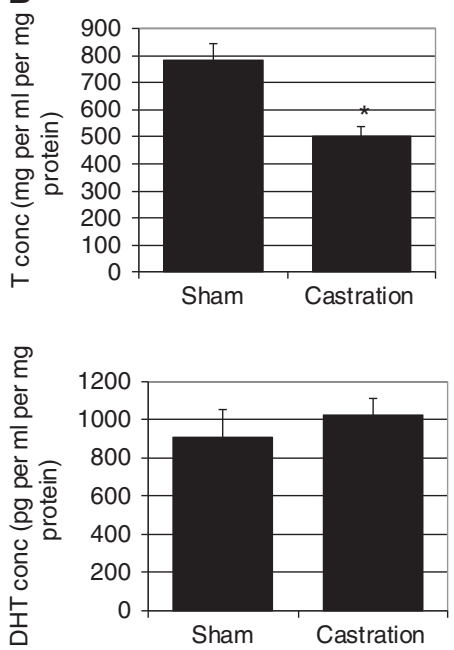

Figure 3. Increased androgen concentrations and androgen biosynthesis enzymes are found in AR-positive Caki2 tumour xenografts. (A) ELISA for serum and intratumoral testosterone was carried out on serum and tumours collected from the castration study using Caki2. In serum, castration decreased $\mathrm{T}$ levels by more than $98 \%$. In tissues, the decrease in T was only $\sim 36 \%$. In addition, tissue T concentration was approximately eightfold higher than the serum level. (B) ELISA for intratumoral T and DHT was performed in the tumours. Intratumoral DHT concentration remained relatively unchanged with castration. *statistically significant.

measured, all six specimens contained levels significantly higher than that found in the Caki2 xenografts (Figure 4B). Specifically, the range of $\mathrm{T}$ and DHT concentrations found in the clinical specimens were 1435-2435 and 2913-4409 $\mathrm{ng} \mathrm{ml}^{-1}$ per mg protein, respectively.

Blocking androgen receptor or androgen biosynthesis inhibits tumour growth. Results of the cell lines and archived clinical specimens suggest a potential therapeutic benefit of targeting androgen signaling in AR-positive RCC. To test this hypothesis, we used the second-generation androgen antagonist, enzalutamide, and CYP17A1 inhibitor AA. Because we have previously reported the cytotoxic effect of enzalutamide in vitro in AR-positive cell lines (Ha et al, 2015), only AA was tested in tissue culture. The results demonstrated that AA significantly decreased the cell count of Caki2 and Hs891.T after 3 days of treatment in a concentrationdependent manner (Supplementary Figure 2). When male mice containing Caki2 xenografts were treated with enzalutamide or AA for 3 weeks, tumour volume at the end of the experimental period was nearly twenty- and nine-fold smaller, respectively, than the 
A

\begin{tabular}{|c|c|c|c|c|c|c|c|}
\hline & CYP11A1 & SRD5A1 & CYP17A1 & HSD17B3 & AKR1C3 & HSD3B1 & HSD3B2 \\
\hline LNCaP & + & ++ & ++ & ++ & ++ & ++ & ++ \\
\hline RCC & - & ++ & + & +++ & ++ & ++ & ++ \\
\hline
\end{tabular}
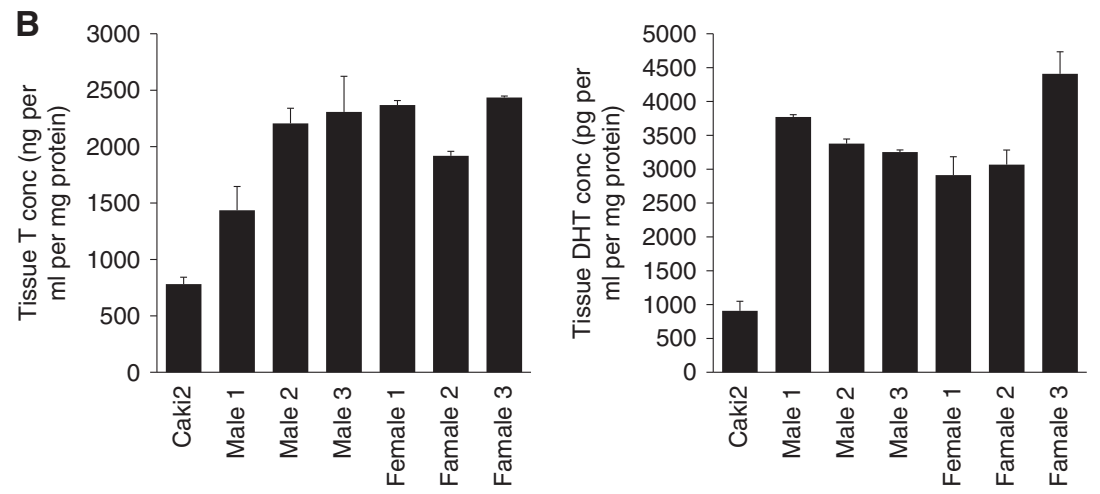

Figure 4. Physiologically significant androgens and androgen biosynthesis enzymes are detected in human female RCC tissues positive for AR. (A) RT-PCR confirmed the presence of the key enzymes required for the intratumoral androgen biosynthesis in human female RCC tissues. LNCaP, the human prostate cancer cell lines, was used as the positive control. (B) ELISA for T and DHT in human RCC tissues obtained from three males and females demonstrated androgen concentrations in the thousands nanogram per mg protein range.
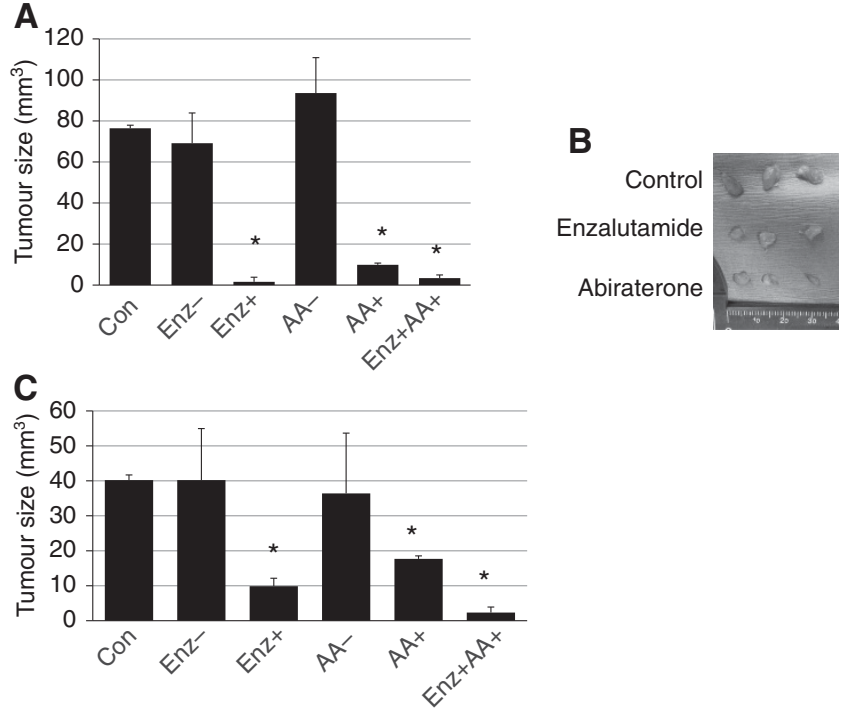

Figure 5. Targeting the androgen signaling axis decreases tumour growth in AR-positive human RCC cell line, Caki2, xenografts. (A) After inoculating the flanks with Caki2, 30 mice were randomized into six groups of five mice each. The treatment was rendered as indicated. Both enzalutamide (Enz) and abiraterone acetate (AA) decreased tumour volume dramatically. (B) Gross picture of tumours collected from mice after treatment. (C) After establishing the tumours, 30 mice were surgically castrated and treated with the indicated agents. Again, Enz and Abi decreased tumour volume significantly. *statistically significant.

vehicle control groups (Figures 5A and B). Further supporting the concept of intracrine androgen synthesis, the inhibitory effect of enzalutamide and AA were observed in mice that were castrated (Figure 5C). When intratumoral androgen concentration was measured after the treatment, AA decreased the tissue level of androgens (Supplementary Figure 3). As expected by being an AR antagonist, enzalutamide treatment group did not show any significant changes in the intratumoral androgen levels.

\section{DISCUSSION}

In the present study, we have demonstrated that RCC cell lines that express AR have the capacity to produce androgens. Importantly, the animal studies have revealed that the androgen signaling axis may potentially be a therapeutic target in patients who have ARpositive RCC. Since the overall rates can be as high as $40 \%$ (Zhu et al, 2014; Ha et al, 2015), the current findings warrant further investigations on the role of androgens in RCC. Interestingly, previous studies have shown that there is no statistically significant difference in rates of AR-positive RCC between males and females (Zhu et al, 2014).

Although the fundamental role of androgens in RCC has been controversial, results of the present study support the notion that $\mathrm{AR}$ is oncogenic and promotes more aggressive features in RCC. Few early pathologic studies have reported an inverse association between stage and AR expression status in RCC (Langner et al, 2004; Zhu et al, 2014; Williams et al, 2015). More recently, Zhao et al. (2016) have examined the TCGA database and have proposed a tumour suppressor function for AR in clear cell RCC. In contrast, we and others have reported an association between poor prognosis and AR expression in RCC (Noh et al, 2013; Ha et al, 2015). This disparity may have resulted from varying methodologies in measuring AR expression levels in RCC tissues. Notwithstanding, functional studies targeting the androgen signaling in RCC cell lines have suggested that AR promotes tumour progression (He et al, 2014; Chen et al, 2015; Ha et al, 2015; Song et al, 2015). Specifically, we have observed that the overexpression of $\mathrm{AR}$ in the AR-negative cell lines increases cellular proliferation in response to androgen treatment ( $\mathrm{Ha}$ et al, 2015). In addition, increased transcriptions of HIF and VEGF have recently been proposed as part of the AR signaling pathway that leads to tumour growth in both in vitro and in vivo models (Chen et al, 2015; Song et al, 2015). The same group also reported that the treatment of AR-positive RCC with ASC-J9, a newly developed AR degradation enhancer, inhibits the growth of RCC tumour in a xenograft study (He et al, 2014). Consistent with these observations, castration slowed the growth of the AR-positive Caki2 xenografts in nude mice. Collectively, the growing body of evidence 
using both tissues and cell lines as well as functional studies suggests that AR promotes rather than suppresses RCC progression.

In addition to the functional studies, the presence of intracrine androgen biosynthesis pathway in AR-positive RCC cells further supports the notion that the androgen signaling axis is protumorigenic in RCC. Specifically, the AR-positive Caki2 and Hs891.T cells were found to have $\mathrm{T}$ and DHT concentrations in the range of $20-70 \%$ of those of the human prostate cancer cell line, LNCaP. In addition, high levels of androgens were also detected in AR-positive human female RCC tissues. Simultaneously, with the exception of CYP11A1, key enzymes necessary for androgen biosynthesis were found in AR-positive RCC cell lines and tissues. Importantly, when the intracellular androgen synthesis was blocked with AA in Caki2 and Hs891.T in tissue culture and tumour xenografts, cellular proliferation was inhibited. Taken together, results of the present study in both in vitro and in vivo models confirm the presence of intratumoral steroidogenesis in AR-positive RCC cells and that intracellular androgen biosynthesis in these ARpositive RCC cells is physiologically significant.

To be precise, the intracellular androgen biosynthesis in ARpositive RCC is not suggestive of a de novo process from cholesterol as the pathway lacks the enzyme CYP11A1, a cytochrome P450scc that catalyzes the side-chain cleavage reaction of cholesterol to pregnenolone (Miller and Auchus, 2011). ARpositive RCC, however, resembles the enzymatic expression patterns of CRPC in the conversion of pregnenolone into more potent androgens, testosterone and DHT. A similar intratumoral androgen metabolism is seen in CRPC where circulating precursors from extra-gonadal sources are converted into $\mathrm{T}$ and DHT, resulting in continued tumour progression in a castrate state (Bluemn and Nelson, 2012). Analogous to CRPC, the intratumoral steroidogenesis in AR-positive RCC is shown in this study to produce sufficient levels of androgens to activate AR even in castrate-like condition. The observed similarity in tissue $\mathrm{T}$ and DHT levels in both male and female, who should have castratelevel of androgen, further support our hypothesis that it is the intratumoral steroidogenesis that is driving the increased tissue androgen levels, rather than the extratumoural sources. These intratumoral steroidogenesis and AR-mediated progression, therefore, may explain the observation that AR expression predicts poor prognosis irrespective of gender. Indeed, it is our speculation that AR-positive RCC behaves similar to CRPC regarding intratumoral androgen metabolism and AR-mediated tumour growth.

The tumour suppressive effects of the 'androgen-deprivation therapy' shown in AR-positive RCC with castration, enzalutamide, and AA have significant clinical implications. First, the continued efficacy of enzalutamide and AA in the castration group confirms that the androgen signaling axis is a potential therapeutic target. Second, the inhibitory effect of AA demonstrates the importance of intracrine androgen synthesis and supports targeting androgen signaling in both males and females. Furthermore, in addition to intracellular inhibition of CYP17 in AR-positive RCC, AA also likely decreases dehydroepiandrosterone sulfate levels, which is converted peripherally into DHT and T. New evidence has also shown that $\Delta^{4}$ abiraterone (D4A), after conversion of AA into this more active form, continues to block multiple steroidogenic enzymes (CYP17A1, $3 \beta \mathrm{HSD}$ and SRD5A1) and even acts as an antagonist to AR (Armstrong and Gao, 2015). On the basis of the current findings, we have recently obtained an industry support to initiate a Phase 0 clinical trial to examine the potential therapeutic effect of enzalutamide in AR-positive RCC. This trial, called BARE (Blocking Androgens in Renal Cell Carcinoma with Enzalutamide), is designed to provide the proof-of-principle for targeting androgens in RCC.

It should be noted that the expression of AR is not a prerequisite for elevated intratumoral androgens. Specifically, increased levels of intratumoral androgens are capable of promoting tumour growth via non-canonical androgen receptors such as glucocorticoid receptor (GR) or G-protein-coupled receptor 6A (GPCR6A) as seen in CRPC (Cleutjens et al, 1997; Pi et al, 2010, 2015; Arora et al, 2013). Further study involving more than 50 cases are underway to verify this concept.

In conclusion, we have demonstrated intratumoral steroidogenesis as a significant source of androgens in AR-positive RCC. The tumour suppressive effects of the anti-androgen therapies in this context suggest androgen signaling as a new potential target of intervention in patients with RCC. We await the results of the BARE clinical trial to start defining the clinical framework in which anti-androgen therapies will be effective in treating RCC.

\section{CONFLICT OF INTEREST}

The authors declare no conflict of interest.

\section{REFERENCES}

Armstrong CM, Gao AC (2015) Drug resistance in castration resistant prostate cancer: resistance mechanisms and emerging treatment strategies. Am J Clin Exp Urol 3(2): 64-76.

Arora VK, Schenkein E, Murali R, Subudhi SK, Wongvipat J, Balbas MD, Shah N, Cai L, Efstathiou E, Logothetis C, Zheng D, Sawyers CL (2013) Glucocorticoid receptor confers resistance to antiandrogens by bypassing androgen receptor blockade. Cell 155(6): 1309-1322.

Bluemn EG, Nelson PS (2012) The androgen/androgen receptor axis in prostate cancer. Curr Opin Oncol 24(3): 251-257.

Chen Y, Sun Y, Rao Q, Xu H, Li L, Chang C (2015) Androgen receptor (AR) suppresses miRNA-145 to promote renal cell carcinoma (RCC) progression independent of VHL status. Oncotarget 6(31): 31203-31215.

Cleutjens CB, Steketee K, van Eekelen CC, van der Korput JA, Brinkmann AO, Trapman J (1997) Both androgen receptor and glucocorticoid receptor are able to induce prostate-specific antigen expression, but differ in their growth-stimulating properties of LNCaP cells. Endocrinology 138(12): 5293-5300.

Ha YS, Lee GT, Modi P, Kwon YS, Ahn H, Kim WJ, Kim IY (2015) Increased expression of androgen receptor mRNA in human renal cell carcinoma cells is associated with poor prognosis in patients with localized renal cell carcinoma. J Urol 194(5): 1441-1448.

He D, Li L, Zhu G, Liang L, Guan Z, Chang L, Chen Y, Yeh S, Chang C (2014) ASC-J9 suppresses renal cell carcinoma progression by targeting an androgen receptor-dependent HIF2alpha/VEGF signaling pathway. Cancer Res 74(16): 4420-4430.

Hirsch MS, Signoretti S, Dal Cin P (2015) Adult renal cell carcinoma: a review of established entities from morphology to molecular genetics. Surg Pathol Clin 8(4): 587-621.

Langner C, Ratschek M, Rehak P, Schips L, Zigeuner R (2004) Steroid hormone receptor expression in renal cell carcinoma: an immunohistochemical analysis of 182 tumors. J Urol 171(2 Pt 1): 611-614.

Lee GT, Jung YS, Ha YS, Kim JH, Kim WJ, Kim IY (2013) Bone morphogenetic protein-6 induces castration resistance in prostate cancer cells through tumor infiltrating macrophages. Cancer Sci 104(8): 1027-1032.

Miller WL, Auchus RJ (2011) The molecular biology, biochemistry, and physiology of human steroidogenesis and its disorders. Endocr Rev 32(1): 81-151.

Mostaghel EA (2013) Steroid hormone synthetic pathways in prostate cancer. Transl Androl Urol 2(3): 212-227.

Noh SJ, Kang MJ, Kim KM, Bae JS, Park HS, Moon WS, Chung MJ, Lee H, Lee DG, Jang KY (2013) Acetylation status of P53 and the expression of DBC1, SIRT1, and androgen receptor are associated with survival in clear cell renal cell carcinoma patients. Pathology 45(6): 574-580.

Payne AH, Hales DB (2004) Overview of steroidogenic enzymes in the pathway from cholesterol to active steroid hormones. Endocr Rev 25(6): 947-970.

Pi M, Kapoor K, Wu Y, Ye R, Senogles SE, Nishimoto SK, Hwang DJ, Miller DD, Narayanan R, Smith JC, Baudry J, Quarles LD (2015) Structural and functional evidence for testosterone activation of GPRC6A in peripheral tissues. Mol Endocrinol 29(12): 1759-1773. 
Pi M, Parrill AL, Quarles LD (2010) GPRC6A mediates the non-genomic effects of steroids. J Biol Chem 285(51): 39953-39964.

Shek D, Tomlinson B, Brown M, Brunson A, Pan CX, Lara PN Jr (2012) Epidemiologic trends in renal cell carcinoma in the cytokine and post-cytokine eras: a registry analysis of 28252 patients. Clin Genitourin Cancer 10(2): 93-98.

Siegel RL, Miller KD, Jemal A (2016) Cancer statistics, 2016. CA Cancer J Clin 66(1): 7-30.

Song W, Li L, He D, Xie H, Chen J, Yeh CR, Chang LS, Yeh S, Chang C (2015) Infiltrating neutrophils promote renal cell carcinoma (RCC) proliferation via modulating androgen receptor $(\mathrm{AR})->\mathrm{c}$-Myc signals. Cancer Lett 368(1): 71-78.

Suzuki K, Nishiyama T, Hara N, Yamana K, Takahashi K, Labrie F (2007) Importance of the intracrine metabolism of adrenal androgens in androgendependent prostate cancer. Prostate Cancer Prostatic Dis 10(3): 301-306.
Williams EM, Higgins JP, Sangoi AR, McKenney JK, Troxell ML (2015) Androgen receptor immunohistochemistry in genitourinary neoplasms. Int Urol Nephrol 47(1): 81-85.

Zhao H, Leppert JT, Peehl DM (2016) A protective role for androgen receptor in clear cell renal cell carcinoma based on mining TCGA data. PLoS One 11(1): e0146505.

Zhu G, Liang L, Li L, Dang Q, Song W, Yeh S, He D, Chang C (2014) The expression and evaluation of androgen receptor in human renal cell carcinoma. Urology 83(2): 510 e519-510 e24.

This work is published under the standard license to publish agreement. After 12 months the work will become freely available and the license terms will switch to a Creative Commons AttributionNonCommercial-Share Alike 4.0 Unported License.

Supplementary Information accompanies this paper on British Journal of Cancer website (http://www.nature.com/bjc) 